\title{
The stimulus range effect: Evidence for top-down control of sensory intensity in audition
}

\author{
SCOTT PARKER \\ American University, Washington, D.C. \\ and \\ BRUCE SCHNEIDER \\ University of Toronto, Mississauga, Ontario, Canada
}

\begin{abstract}
The influence of intensity range on the perceived magnitude of a stimulus is well documented and usually attributed to response biases. Recent studies, however, have suggested that the range effect might be sensory in origin. To test this notion, we had one set of subjects compare loudness intervals in three conditions: a broad-range condition (15 tones, 23-95 dB SPL), a soft shortrange condition (the lowest 10 tones from the broad-range condition), and a loud short-range condition (the highest 10 tones). Nonmetric scaling showed that the broad-range and loud short-range conditions had identical loudness functions. However, the second derivative of the loudness function was larger for the soft short-range condition than for the broad-range condition. This pattern of results is consistent with the notion of a nonlinear amplifier whose gain and degree of nonlinearity are adjusted under top-down control, so as to prevent distortion and increase discriminability.
\end{abstract}

It is well known that measures of sensory events are subject to context effects. For example, numerical estimates of loudness are affected not only by stimulus intensity, but also by the context provided by the instructions, and by the number, spacing, and range of the sounds being presented. Many investigators interested in sensory processes tend to regard these context effects as "nuisance" variables and would like to banish them to the realm of higher order, nonsensory processing, where they can be catalogued, controlled for, and ultimately dismissed. For example, S. S. Stevens $(1957,1971)$ argued that it often was difficult to uncover the "true" sensory representation because of the operation of a number of "psychological" factors that bias subjects' reports of sensory magnitude. Indeed, response biases have been invoked to explain why varying the stimulus range (Teghtsoonian, 1973), stimulus spacing (Stevens \& Galanter, 1957), and instructional parameters (Robinson, 1976) perturb the form of the loudness function, and many schemes have been developed to control, counterbalance, and/or circumvent these so-called higher order effects. For example, Schneider, Parker, Valenti, Farrell, and Kanow (1978) used nonmetric scaling techniques to circumvent the operation of many of the response biases found in nu-

This research was supported by a grant from the Natural Sciences and Engineering Research Council of Canada. We thank Jane Carey for conducting these experiments. Correspondence should be addressed to either S. Parker, Department of Psychology, American University, Washington, DC 20016-8062, or B. Schneider, Department of Psychology, Erindale Campus, University of Toronto, Mississauga, ON L5L 1C6 Canada. merical estimation techniques, and Zwislocki and Goodman (1980) have developed a scaling technique that they believe is capable of overcoming these response biases. Such viewpoints and approaches, until recently, reflected a consensus that most, if not all, of the above-mentioned context effects were due to the operation of higher level, nonsensory processes, and that these "cognitive" factors interfered with the measurement of relatively simple sensory processes.

Recent experiments by Marks and his colleagues (Algom \& Marks, 1990; Marks, 1988; Marks, Szczesiul, \& Ohlott, 1986; Marks \& Warner, 1991) have identified a context effect in loudness judgments that may not be attributable to such higher order processing. Marks (1988) had subjects estimate the loudnesses of both 500- and $2500-\mathrm{Hz}$ tones, intermixed in the same session, by using the method of magnitude estimation. In one condition, the sound-pressure levels (SPLs) of the $500-\mathrm{Hz}$ tones ranged from 35 to $75 \mathrm{~dB}$, and the $2500-\mathrm{Hz}$ tones ranged from 50 to $85 \mathrm{~dB}$. In the other condition, the $500-\mathrm{Hz}$ tones ranged from 55 to $90 \mathrm{~dB}$, and the $2500-\mathrm{Hz}$ tones ranged from 30 to $65 \mathrm{~dB}$. Because both the 500 - and $2500-\mathrm{Hz}$ tones were intermixed within a session, it was possible to determine, for example, the intensity of the $2500-\mathrm{Hz}$ tone whose loudness judgment equaled that given to a 70$\mathrm{dB}, 500-\mathrm{Hz}$ tone. In Condition $1, \mathrm{a} 68-\mathrm{dB}, 2500-\mathrm{Hz}$ tone was rated as being as loud as the $70-\mathrm{dB}, 500-\mathrm{Hz}$ tone. However, in Condition 2, a $51-\mathrm{dB}, 2500-\mathrm{Hz}$ tone was rated as being as loud as the $70-\mathrm{dB}, 500-\mathrm{Hz}$ tone, indicating a 17-dB shift in the loudness match between the two conditions. The major difference between the two conditions was that, in Condition 1, the intensity levels of the 
$500-\mathrm{Hz}$ tones were lower than those of the $2500-\mathrm{Hz}$ tones, whereas the reverse was true in Condition 2. It appears that reversing the intensity ranges at the two frequencies dramatically affected the loudness matches. Thus, loudness matches obtained from a magnitude estimation procedure are affected by relations between the ranges of the sounds at the two different frequencies.

Because magnitude estimation was involved, initially it was tempting to assign the cause of this context effect to the malleability of people's use of numbers, as Marks (1988) did. Subsequent studies of this context effect, however, began to indicate that the source of the effect might be sensory rather than cognitive. Schneider and Parker (1990) investigated the context effect found by Marks by having subjects choose which of two pairs of tones defined the larger loudness interval. The tones within a pair could differ in both intensity and frequency $(500$ or $2500 \mathrm{~Hz}$ ). Nonmetric scaling techniques were used to determine loudness values for both the 500 - and $2500-\mathrm{Hz}$ tones, making it possible to find, for example, the decibel value of a $2500-\mathrm{Hz}$ tone that was as loud as a $70-\mathrm{dB}, 500-\mathrm{Hz}$ tone. In different conditions, the intensity range at one frequency was varied relative to the intensity range at the other frequency. Contrary to Schneider and Parker's expectations, the relative intensity ranges at the two frequencies did affect the derived loudness matches in some conditions. Because their subjects did not make numerical judgments, these context effects could not readily be attributed to numerical response biases.

Other evidence suggesting a sensory origin for the context effect has been provided by Marks and Warner (1991), and Marks (1992). Marks and Warner, using Marks's (1988) paradigm, varied the frequency separation between the two groups of tones and found that frequency separation governed the size of the context effect. When the frequency separation was less than a critical band, so that the auditory filter that was centered on one frequency would still respond to stimuli at the other frequency, the context effect disappeared. Thus, the context effect is not simply due to the discriminability of the two frequencies; it also requires that the two frequencies be processed in separate sensory channels. If the context effect depends on the critical band-clearly a peripheral process-it is difficult to attribute its occurrence to cognitive rather than sensory processing. Finally, Marks (1992) occasionally interrupted magnitude estimation sessions employing his (1988) context paradigm to ask the subjects which of two stimuli was louder (a $500-\mathrm{Hz}$ or a $2500-\mathrm{Hz}$ tone). Note that this judgment did not require a numerical response, only a binary decision by the subject; yet he found that the subjects' loudness comparisons were affected by the stimulus context. Again, this suggests a sensory origin for the context effect.

If the context effect has a sensory origin, what sensory process produces it? Schneider and Parker (1990) suggested that the context effect is produced by the operation of a narrow-band amplifier, which is engaged when all the stimuli within its bandwidth are soft. In their experiments, when soft tones at one frequency (f1) were combined with a broad range of tones at another frequency (f2), the loudnesses of at least some of the f1 tones were increased. When, however, only loud tones at $f 1$ were combined with a broad range of tones at $f 2$, no change in the loudnesses of the $\mathrm{f} 1$ tones was observed. These results are consistent with the notion that context influences the "gain"' in the critical band around $f 1$. If, during the course of the experiment, subjects hear only soft $\mathrm{f} 1$ tones, they can "turn up" the gain in this channel in order to process the tones more readily. If, on the other hand, the f1 tones are sometimes loud, then turning up the gain of the fl amplifier might be expected to produce distortion in that channel when the louder tones are presented. Therefore, the gain would be turned up only when no loud tones are expected. This model requires that the stimuli at two frequencies be separated by at least one critical band, as Marks and Warner (1991) found. If the $\mathrm{f} 2$ tones "spilled over" into the $\mathrm{fl}$ channel, then the $\mathrm{fl}$ channel would experience both loud and soft stimuli, so that no context effect would be observed.

Of course, rather than turning up the gain on soft tones, we could hypothesize that the gain is normally set high but is turned down in the presence of loud sounds, and that the mechanism functions more as an attenuator than as an amplifier. Either an attenuator or an amplifier could provide the kind of gain control that would account for the results found by Schneider and Parker (1990). For simplicity, we refer to the gain control mechanism as an amplifier.

Note that the notion of an internal amplifier does not require the presence of tones at two different frequencies in order for stimulus range to have an effect on the coding of loudness. If only soft tones at one frequency are presented, then subjects should turn up the gain in that channel, independent of anything that is happening in other channels. Therefore, the context effect should occur in experiments in which tones of one frequency are used. One way to demonstrate the existence of this effect is to compare the loudnesses of a set of soft tones, when only they are presented (Condition S), with their loudnesses when they are combined with loud tones to produce a set of tones covering a broad range (Condition B). Only in Condition $\mathrm{S}$ should the amplifier be engaged, thereby increasing the loudnesses of the soft tones. However, if we were to compare the loudnesses of a set of loud tones when only they were presented (Condition L), with the loudnesses when they were included within a broad range (Condition B), we would not expect any change in their loudnesses because the amplifier would not be engaged in either condition. As in our previous experiments, we used a nonmetric analysis of comparisons of loudness intervals to circumvent any response biases in numerical judgments, so that any context effect that remained could not easily be attributed to higher order processes.

\section{METHOD}

\section{Subjects}

Eight subjects, students and research assistants associated with the Psychology Department of the University of Toronto, partici- 
pated in these experiments. There were 5 males and 3 females, ranging in age from 25 to 37 years. All were paid for their participation and reported having no auditory pathology.

\section{Apparatus}

The 1-kHz tones were generated digitally (sampling rate of $20 \mathrm{kHz}$ ) and converted to analog form by using a Spectrum TMS3200C25 16-bit digital signal processing board in a Comptech 386 computer. Calibration using a Wavetek spectrum analyzer and an oscilloscope showed that all harmonics were at least $80 \mathrm{~dB}$ below the highest signal level and $27 \mathrm{~dB}$ below the lowest signal level ( $23 \mathrm{~dB}$ SPL). The amplitude of the signal was controlled digitally, and the signals were switched on and off with a $10-\mathrm{msec}$ rise and fall time.

Stimuli were presented monaurally (preferred ear) over TDH49 earphones. The earphones were calibrated by using a Brüel \& Kjaer 2209 sound-level meter with a 1-in. microphone in a $6-\mathrm{cm}^{3}$ coupler. The subjects sat in a double-walled sound-attenuating chamber.

\section{Procedure}

The broad-range set (Condition B) contained 15 stimuli, whereas the short-range soft set (Condition S) and the short-range loud set (Condition L) contained only 10 stimuli each. Five stimuli were common to all three sets. The stimuli in Condition B were 23, 29, 34, $40,46,51,56,62,67,70,75,79,84,90$, and $95 \mathrm{~dB}$ SPL. The stimuli in Condition $S$ were the lower 10 of those in Condition B (23-70 dB SPL); the stimuli in Condition L were the higher 10 of those in Condition B (51-95 dB SPL). Thus, stimulus levels of 51, $56,62,67$, and $70 \mathrm{~dB}$ SPL were common to all three conditions.

Each pair of distinct stimuli defined a loudness interval. In both the soft and loud short-range conditions there were $45(10 \times 9 / 2)$ distinct stimulus pairs possible from the 10 intensities. Thus, the number of distinct comparisons among these $\mathbf{4 5}$ loudness intervals was $45 \times 44 / 2=990$. However, because loudness is monotonic with intensity, we do not need to compare loudness intervals in which the tone intensities defining one interval bracket those defining the other interval. For example, we know that the loudness interval defined by tonal intensities of 50 and $75 \mathrm{~dB}$ is larger than that defined by intensities of 55 and $70 \mathrm{~dB}$, because the first interval brackets the second. Removing these bracketed cases reduces the number of comparisons to 540 (Bissett \& Schneider, 1991, p. 694; Fagot, 1959, pp. 165-166).

The 15 stimuli in the broad-range condition result in 105 $(15 \times 14 / 2)$ distinct stimulus pairs. Of the $5,460(105 \times 104 / 2)$ possible loudness-interval comparisons among these pairs, we can eliminate 2,275 of them because the intensities of one pair bracket those of another, leaving 3,185 loudness-interval comparisons to be tested (Bissett \& Schneider, 1991).

Because the remaining number of comparisons in all three conditions was so large, the subjects were presented with a random subset of these comparisons. Bissett and Schneider (1991) showed that loudness scales constructed from only $30 \%$ of 540 critical comparisons among 10 tones recover the "true" form of the loudness scale almost as well as when they are constructed from all 540 comparisons. Similarly, when there are 15 stimuli, a mere $20 \%$ of the 3,185 critical comparisons are sufficient. Thus, in the present experiment we used only 162 comparisons in the loud and soft shortrange conditions and only 637 comparisons in the broad-range condition. The subset of comparisons (162 or 637) chosen in each condition was randomly selected. In this random selection, the stimuli were labeled 1-10 for the soft and loud short-range conditions, and the 540 interval comparisons were defined by listing their component stimuli (e.g., 1,3 vs. 2,7 defined an interval comparison). From this set of interval comparisons, 162 were chosen at random by a computer program. The same 162 comparisons were used for both the loud and soft short-range conditions. The only differences were that Stimuli $1,2, \ldots, 10$ in the soft short-range condition had in- tensities of $23,29, \ldots, 70 \mathrm{~dB}$, whereas Stimuli $1,2, \ldots, 10$ in the loud short-range condition had intensities of $51,56, \ldots, 95 \mathrm{~dB}$. In the broad-range condition, the stimuli were labeled $1-15$, the interval comparisons were defined, and 637 of them were chosen at random. In each condition, all the subjects were presented with the same comparisons. However, each subject was presented with the interval comparisons for a given condition in a different random sequence.

Within a single pair, there were two possible orders in which the tones could occur; there were two pairs, so there were four possible orders of tones within pairs. In addition, each pair could be assigned to either Button 1 or Button 2, so there were eight possible arrangements. Each of the eight arrangements for each comparison of pairs was randomly assigned to 1 subject.

Both the loud and soft short-range conditions (Conditions $\mathrm{L}$ and S) consisted of 4 sessions: a practice session, which contained 50 comparisons, and 3 experimental sessions. The purpose of the practice session was to familiarize the subjects with the range of stimuli employed. The first 2 experimental sessions contained 60 comparisons each, and the third contained 42 comparisons. Within each experimental session, the subject experienced the full range of intensities, usually within 3 to 4 comparisons. The broad-range condition consisted of 13 sessions. The first was a practice session containing 50 comparisons. The next 8 contained 60 comparisons each. Because there was a 1-month break between the 8th and 9th sessions, the 9th session was preceded by another practice session containing 50 comparisons. Experimental Sessions 9 and 10 contained 60 comparisons, and Session 11 contained 37 comparisons. Again, each subject experienced the full range of intensities within each experimental session, usually within 5 to 6 comparisions.

In each condition, stimulus presentation was initiated when the subject pressed one of two buttons. When Button 1 was pressed, the computer, after a 50-msec delay, presented the two intensities in Pair 1 for $750 \mathrm{msec}$ each, separated by a 500 -msec silent interval. Pressing Button 2 presented the second pair of tones in an identical manner. The subjects were allowed to listen to each pair as many times as they wished before indicating their judgment by pressing one of the two response buttons. They were instructed to listen to the loudness difference between the tones in each pair, and indicate the pair in which the tones were more widely separated in loudness.

\section{RESULTS}

If 5 or more of the 8 subjects judged pair $(i, j)$ to differ more in loudness than pair $(k, l)$, we would consider the loudness interval in the first pair to be greater than the loudness interval in the second pair [i.e., $(i, j)>(k, l)$ ]. If only 4 subjects judged the first as being larger than the second, we would consider the loudness-difference comparison to be indeterminate [i.e., $(i, j) \approx(k, l)]$. The number of such indeterminate pairs was 51 in the broad range, 17 in the soft short range, and 4 in the loud short range.

To determine a loudness scale for each condition, a nonmetric scaling procedure specifically designed for comparisons of intervals (Bissett \& Schneider, 1991) was employed. In this procedure, loudness values, $L$, are assigned to each of the tones, so that whenever

$$
(i, j)>(k, l) \text {, then }|L(i)-L(j)|>|L(k)-L(l)| \text {. }
$$

Of course in data with error, it is not possible to do this perfectly; hence the program minimizes a badnessof-fit index called $G$, where $G$ is the proportion of times 
that relation 1 is violated. If $G=0$, then the loudnessdifference comparisons are perfectly predicted by the difference in loudness values. Values of $G$ were .084 for Condition B, .090 for Condition S, and .044 for Condition L.

These values of $G$ can be used to estimate the degree to which the loudness values represent interval scale measurement. To accomplish this, the index of coordinate metric recovery $(\mathrm{CM})$ was estimated. $\mathrm{CM}$ is the squared Pearson correlation coefficient between the true loudness values of the stimuli (which presumably generated the obtained comparisons) and the loudness values produced by the program. Hence, $\mathrm{CM}$ varies between 0 and 1 , and $\mathrm{CM}=1$ indicates that the true loudness values have been perfectly recovered. In empirical investigations conducted with these techniques, the true loudness values are unknown. Bissett and Schneider (1991), however, have shown how CM can be estimated from $G$. Hence, if the estimated value of CM is sufficiently high, the loudness values obtained from the program can properly be regarded as representing interval scale measurement. The estimated value of CM was greater than .99 in all three conditions. Given these high values, the loudness values in each condition can be considered as representing interval scale measurement of loudness.

The loudness values determined in this fashion are unique only up to affine transformation-that is, up to the addition of a constant and multiplication by a constant. If, for example, the loudness values determined from the nonmetric program were all doubled and then increased by 20 , the differences among these new values would still predict the subjects' judgments precisely as well. Therefore, in order to compare loudness values across conditions, we first have to normalize them. Recall that five stimuli were common to all three conditions. Standard least squares linear regression techniques were used to determine estimates of the values of $\alpha$ and $\beta$ in the equation $y=\alpha y^{\prime}+\beta$, where $y^{\prime}$ represents the loudnesses of the five tones in Condition $S$ that were also common to Condition B, and $y$ represents the loudness of those Condition B tones. All of the tones in Condition S were then rescaled by using the least squares estimators of $\alpha$ and $\beta$. A similar procedure was followed in order to rescale the loudness values from Condition $L$. The correlation coefficients for these five values were .929 for Conditions B and L, and .980 for Conditions B and S.

If the relation between loudness and sound-pressure level is independent of the physical range of the stimulus sets employed, the loudness values from all three conditions should lie along a single curve when plotted as a function of sound pressure. In particular, if loudness is a power function of intensity, and the loudness values, $L_{v}$, determined in these conditions are affine transformations of loudness, then

$$
L_{\mathrm{v}}=a p^{n}+b,
$$

where $p$ is sound-pressure level, $a$ and $b$ are constants, and $n$ is the exponent of the power function. These loudness values (in arbitrary units) are plotted in Figure 1 as a function of decibels of sound pressure (dB SPL). The smooth curve drawn through the data points is the least squares fit of Equation 2 to the loudness values from the broad-range experiment, and it has an exponent (as a function of sound pressure) of 0.35 . Note that the loudness values for the broad-range Condition $B$ are nearly identical to those from the loud short-range Condition $\mathrm{L}$. However, the five softest loudness values from the soft short-range Condition S diverge from those of the broadrange condition. Note that this divergence occurs despite the fact that the five loudest of the soft short-range loudness values were adjusted so that they were as close as possible to the equivalent five values in the broad-range condition. This suggests that the function that relates loudness to sound-pressure level in Condition $S$ differs from the functions for Conditions B and L, but that the functions for the latter two conditions are equivalent.

The loudness values obtained in Condition $S$ are plotted as a function of the equivalent values in the broad-range condition (Condition B) in Figure 2. Also plotted are the loudness values from Condition $L$ as a function of their corresponding values in Condition B. Here, a point represents the loudness values of a tonal intensity that was common to both conditions. Loudness values for the soft short-range condition are a positively accelerated function of their broad-range counterparts. On the other hand, loudness values for the loud short-range condition appear as a straight line against their broad-range counterparts.

Similar plots were examined for the individual subjects. To test for positive acceleration in these plots, power functions of the form

$$
y=\alpha x^{r}+\beta
$$

were fit to each plot, where $x$ represents the broad-range projection values, $y$ represents their short-range counterparts, and $\alpha, \beta$, and $r$ are fitted constants. (Before fitting these functions to the data of individual subjects, each set of projection values was normalized so that they ranged from 0 to 1.0.) Values of $r$ greater than 1 indicate the presence of positive acceleration in a plot. ${ }^{1}$ For all 8 subjects, the values of $r$ were greater than 1 for the soft shortrange versus broad-range plots $(r=1.74,2.28,2.22$, $2.57,1.25,1.20,2.38$, and 1.42 for Subjects 1-8, respectively). If, in the population, both sets of projection values are linearly related, we would expect an exponent of 1.0 . A sign test, used to verify the hypothesis that the median value of the exponent is 1.0 , was significant at the .01 level (two-tailed). On the other hand, for the loud shortrange versus broad-range plots, the values of $r$ were .87 , $.83, .86,1.15, .77,1.43, .81$, and 1.11 for Subjects $1-8$, respectively. The average exponent (.98) is very close to 1.0. A sign test of the hypothesis was not significant $(p>$ .70 ). Thus, there is no evidence that the loudness projections of the individual subjects deviate systematically from a linear relation when the loud short-range values are plotted as a function of their broad-range counterparts. We conclude, as indicated in Figure 2, that (1) the loudness values for the soft tones in the soft short-range condition are a positively accelerated function of the loud- 


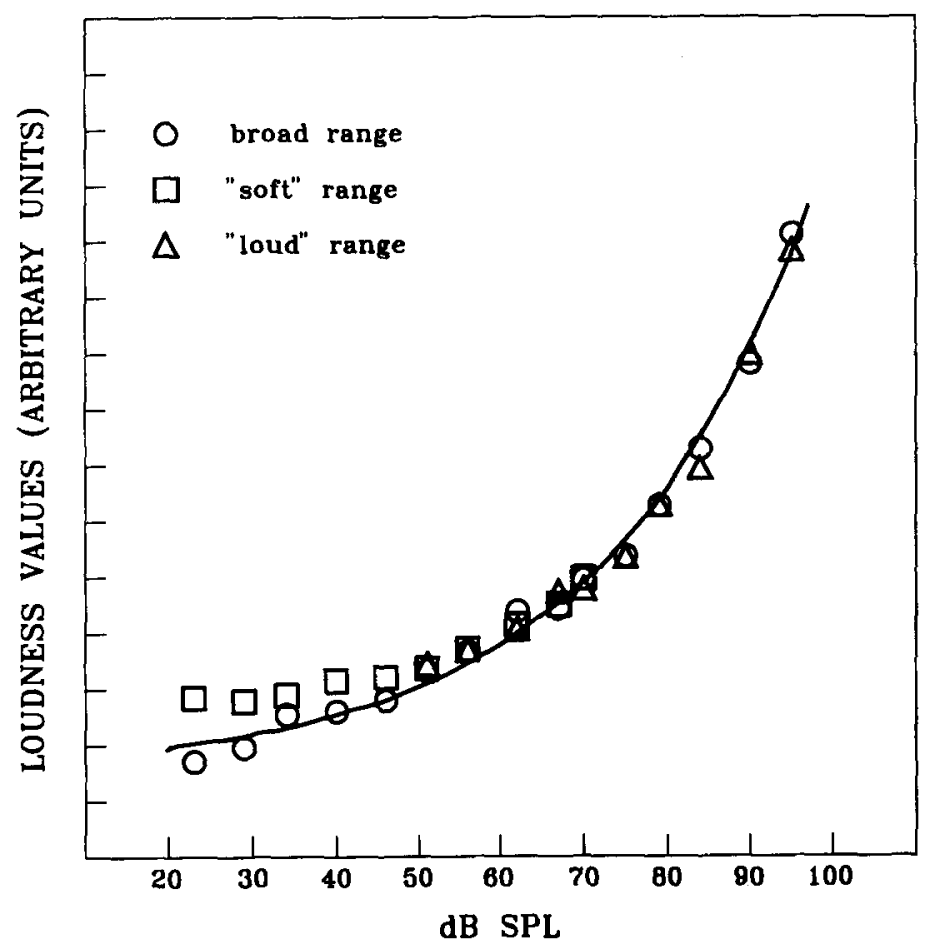

Figure 1. Normalized loudness projections as a function of sound-pressure level (SPL) for tones in all three conditions. The smooth curve is a power function of sound pressure (Equation 2) with an exponent of 0.35 .

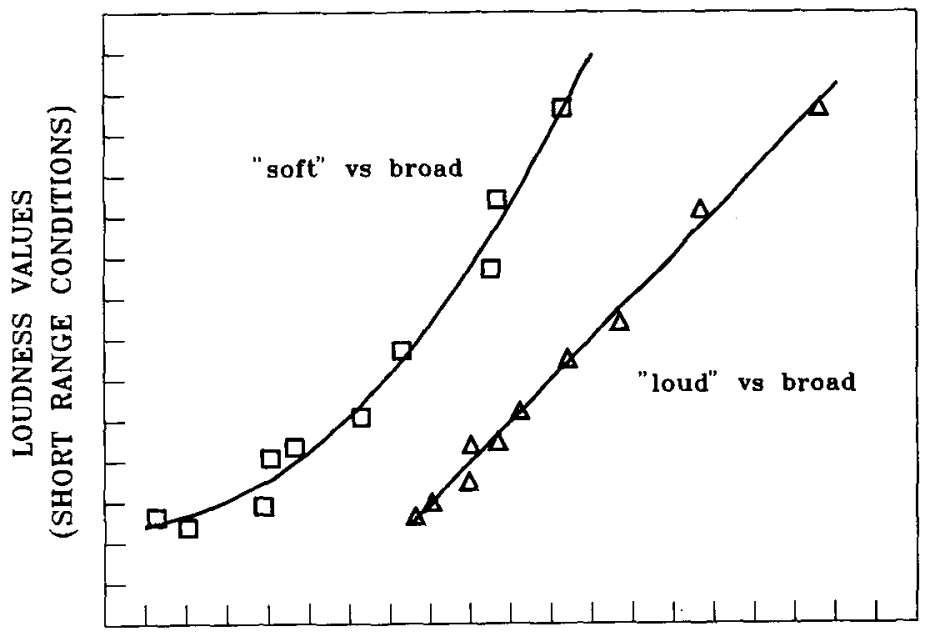

LOUDNESS VALUES (FULL RANGE CONDITION)

Figure 2. Loudness projections for stimuli in the two short-range conditions plotted against the loudness projections for the same stimuli in the broad-range condition. For clarity, all four sets of loudness projections have been adjusted to have the same mean and range, and the function for loud short-range versus broad range has been shifted to the right. The smooth curve fit to the squares (soft vs. broad) is a power function with an exponent of 1.8, reflecting the significant positive acceleration (see text). A straight line (a power function with an exponent of 1.0 ) provides a good fit to the triangles (loud vs. broad). 
ness values for the same tones in the broad-range condition, and (2) the loudness values for the loud short-range tones are linearly related to the loudness values of the same tones in the broad-range condition.

\section{DISCUSSION}

\section{Stimulus Range and the Loudness Function}

A number of studies (e.g., Poulton, 1968; Teghtsoonian, 1973) have shown that the range of stimuli affects the form of the loudness function when direct scaling techniques (magnitude estimation, magnitude production, etc.) are employed. If loudness in a magnitude estimation experiment is described as a power function of sound intensity, then an increase in stimulus range produces a decrease in the exponent of the power function (Teghtsoonian, 1973). Teghtsoonian accounts for this range effect by postulating that the range effect is a consequence of the so-called regression effect that is found in direct scaling techniques. Stevens and Greenbaum (1966) noted that the size of the exponent obtained in a magnitude estimation experiment, in which subjects assign numbers to represent the magnitudes of the stimuli, was typically smaller than that obtained in a magnitude production experiment, in which subjects adjust stimuli to produce specific sensation magnitudes. Stevens and Greenbaum argued that subjects in any matching experiment (e.g., matching numbers to loudnesses or matching loudnesses to numbers) were reluctant to use a large range, whatever the variable (number or loudness) under their control. Thus, in a magnitude estimation experiment with a broad stimulus range, the numerical range would be constricted, whereas in a magnitude production experiment with a broad range of numbers, the range of tonal intensities produced would be constricted.

This reluctance to use a broad range in a magnitude estimation experiment would have a more severe effect on responding as the stimulus range becomes broader. Consequently, when the stimulus range is small, the corresponding range of subjective magnitudes will be small. Therefore, subjects would be under no pressure to restrict the range of the variable under their control (number), and the exponent of the underlying power function would not be affected. However, when the stimulus range is large, restriction of the response range would reduce the size of the power function exponent. Therefore, the range effect could be attributed to higher order response biases that are unrelated to sensory processing.

Note that if the range effect is due to a response-range constriction or to any other sort of constraint on the subject's response system, then we would not expect to find a range effect in the present experiment. In the present experiment, the subjects simply reported which of two loudness intervals was the larger. When subjects are asked to describe how they performed this task, they usually say something to the effect that they had judged whether the (loudness) difference in the first pair was larger than the difference in the second pair. The subjects in the present study denied that they had assigned numerical values to the individual tones and/or to the loudness difference between tones in arriving at their binary choice. Because this was a nonnumerical binary judgment, any restrictions on the response range that may affect the form of the loudness function in direct scaling techniques simply do not apply. Therefore, we would not expect to find a range effect if it is due to a response-range constriction or to some sort of response bias. Indeed, there is no range effect when a comparison is made between a short loud range and a broad range (see Figures 1 and 2). Magnitude estimation experiments, however, do produce strong range effects when loud stimuli (such as those included in Conditions L and B) are employed (Teghtsoonian, 1973). Thus, if we were to compare only Conditions $L$ and $\mathrm{B}$, we would conclude that, once response biases are eliminated through the use of nonmetric scaling techniques, the range effect disappears.

The present experiment, however, reveals an asymmetry in the range effect. When a short soft range is compared with a broad range that includes the soft stimuli, the rate of growth of loudness (the second derivative of the loudness function) is greater for a set of stimuli-all of which are soft-than it is for the same soft stimuli when they are included in a set containing loud stimuli. This is indicated most clearly in Figure 2, which shows that the loudness values for the set of soft stimuli are a positively accelerated function of the loudness values of the same stimuli when they are part of the broad-range condition. Within the present paradigm, the only way that such results could be obtained is if the inclusion of loud stimuli in a condition reversed the direction of some of the loudness-interval comparisons conducted entirely within the set of soft stimuli. Thus, the inclusion of loud stimuli within a set of comparisons, as in Condition B, changes the rank order of loudness intervals among the soft stimuli. It is difficult to see how any constriction placed on a response range could produce such an effect. Moreover, if for some reason response biases were responsible for changing the rank order of loudness intervals among the soft stimuli, then why were they not capable of doing so when a loud short-range set (Condition L) was broadened by adding soft tones to it in order to produce a broad-range set (Condition B)? We conclude, therefore, that the range effect found in this experiment shows that the loudness intervals among a set of soft stimuli are changed by adding loud stimuli to the set, whereas the loudness intervals among a set of loud stimuli are unaffected by the addition of soft stimuli to the set.

It is worth noting that if we assume that Teghtsoonian's (1973) model of intramodality range effects characterizes the sensory representation of the stimuli, we would not expect an asymmetric range effect. Teghtsoonian was able to show, for a number of different sensory continua, that 
the observed exponent in a direct scaling experiment was inversely proportional to the logarithm of stimulus range. That is,

$$
n_{0}=n+\frac{c}{\log \theta_{\mathbf{r}}},
$$

where $n_{0}$ is the observed exponent, $\theta_{\mathrm{r}}$ is the ratio of the highest to lowest stimulus intensity in the set, and $n$ and $c$ are constants. If this equation specifies the exponent of the power function that maps stimulus intensity into sensory magnitude, then we would expect nearly equivalent exponents in the loud and soft short-range conditions because they have nearly equivalent logarithmic ranges ( 47 and $45 \mathrm{~dB}$ for Conditions $S$ and $\mathrm{L}$, respectively). Therefore, we would expect the same increase in exponent in both Conditions $\mathrm{S}$ and $\mathrm{L}$ relative to the exponent in Condition $B$. This means that the same positive acceleration in the function relating the loudness values in Condition $S$ to those in Condition B should also relate the loudness values in Condition $L$ to those in Condition $B$, if the effect found here proved to be the same as that found in direct scaling experiments. The fact that there is positive acceleration in Conditions $S$ versus $B$, but not in Conditions $L$ versus $B$, suggests that log stimulus range per se is not responsible for this asymmetry.

It is important to note that the results of the present experiment do not deny the existence of response-range constraints in direct scaling techniques. Indeed, the lack of any range effect for the loud short-range versus the broadrange stimulus sets in the present experiment, as well as the obvious presence of a range effect for loud stimuli in direct scaling experiments (see Teghtsoonian, 1973), clearly argues for the existence of response-range constraints and other forms of response bias in direct scaling experiments. The present results argue that the range effects found in direct scaling experiments are not entirely due to a change in the function that relates responses to perceptual magnitudes.

The results of the present study as well as those from Schneider and Parker (1990) are consistent with the notion that there is a nonlinear amplifier in the auditory processing stream whose gain is subject to top-down control. When the stimulus set contains some high-intensity tones, no amplification is applied to the tone set. Therefore, in experiments that include loud tones, the form of the loudness function should be independent of the range of the stimuli, provided that there are no response-bias effects. The loudness values obtained from two other studies employing nonmetric comparisons of loudness intervals, along with those from the broad-range condition and the loud short-range condition, are plotted in Figure 3. The other two studies (Schneider, 1980; Schneider, Parker, $\&$ Stein, 1974) are based on the group data from 5 and 8 subjects, respectively, and employ a range of stimuli that differs from Conditions L and B. Also, because they were conducted by using the same nonmetric scaling techniques, they should not be subject to response-bias effects. Therefore, we would expect the form of the loudness function in these four experiments to be equivalent because they all employ high-intensity tones. Figure 3 indicates a high degree of agreement in the form of the loudness function. The smooth curve drawn through the points is a power function of sound pressure fit to the data of Conditions $L$ and $B$, with an exponent of .35. The data points from the other two experiments are well described by the same power function. Figure 3 indicates that when response biases are removed and the stimulus set contains some high-intensity tones, the loudness function is not affected by the stimulus range. ${ }^{2}$

\section{A Narrow-Band, Nonlinear Amplifier}

Although the results of Schneider and Parker's (1990) experiment suggested the existence of a narrow-band amplifier that was turned on when all of the stimuli within a critical band were soft, it was not possible to determine whether the amplifier was linear or nonlinear. A linear amplifier, however, cannot account for the results of the present experiment. To see why this is the case, suppose, as in Equation 2, that the loudness values are a linear function of sound pressure raised to a power. A linear amplifier would simply multiply all of the sound pressures by a constant, $k$, so that the loudness values, after amplification, $L_{\mathrm{v}}^{\prime}$ would be

$$
L_{v}^{\prime}=a(k p)^{n}+b=a^{\prime} p^{n}+b, \text { where } a^{\prime}=a k^{n} .
$$

Therefore, the loudness projections obtained after linear amplification would be linearly related to the loudness projections before amplification, and the nonlinear relation shown in Figure 2 between the loudness values for the soft tones in Condition S and their soft counterparts in the broad-range condition would not have been found. We conclude that, if there is an amplifier, it must be nonlinear.

If there is an amplifier, what function does it serve and why is it nonlinear? In circuit designs, amplifiers are employed to boost signal intensity when signal amplitude is too low for adequate signal processing. Suppose, for example, that the processing task is to discriminate between two signals, but that the circuit that performs the discrimination is somewhat noisy. If the amplitudes of the two signals, at the point of input to the discrimination circuit, are so low that they are all but obscured by the internal noise in the discrimination circuit, the obvious remedy is to amplify the two signals before they are sent to the discrimination circuit in order to improve the signal-tonoise ratio. Amplification changes may also be required when signal amplitudes are too high. Suppose that the discrimination circuit, in addition to being noisy, begins to peak-clip signals above $100 \mathrm{mV}$. If the two signals to be discriminated are 500 and $700 \mathrm{mV}$, respectively, they would be difficult to discriminate if they were peakclipped at $100 \mathrm{mV}$. If, however, they were attenuated by $20 \mathrm{~dB}$, they would be relatively easy to discriminate. Therefore, with regard to circuit design, amplifiers are handy components if signal amplitudes vary widely and if discrimination circuits are noisy and have limited response ranges. There can be little doubt that the neural networks that discriminate among auditory stimuli are likely to be noisy and to have a limited response range. 


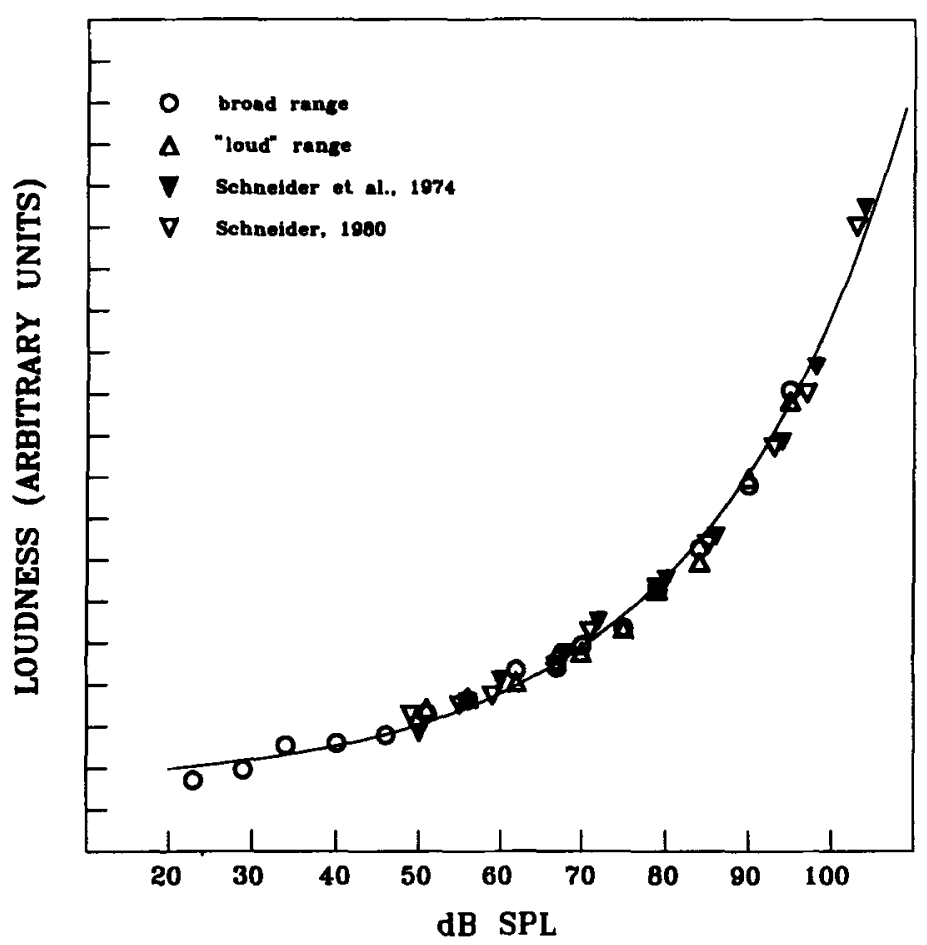

Figure 3. Loudness values from the broad-range and loud short-range conditions are shown as a function of sound-pressure level (SPL) along with loudness projections from two other experiments. The smooth curve is a power function of sound pressure with an exponent of 0.35 .

Therefore, it would not be unreasonable to find that amplification and/or attenuation (we will consider them as equivalent) occur at various points in the signal-processing stream. Indeed, negative feedback models that nonlinearly change the visual system's gain have been used to account for certain aspects of light adaptation (Fuortes \& Hodgkin, 1964; Marks, 1972; Sperling \& Sondhi, 1968), and negative feedback loops (via olivocochlear feedback) have been proposed in audition (e.g., Geisler, 1974; Kim, 1984) to extend the dynamic range of auditory nerve fibers.

The results of the present experiment suggest that the amplification is nonlinear. Again, considering a circuit design, under what set of circumstances would it be reasonable to employ a nonlinear amplifier? Figure 4 shows a circuit with a nonlinear amplifier, where the nonlinearity is a result of a negative feedback loop. It is assumed that the input to the amplifier is a sinusoidal signal, $A \cos \left(2 \pi f_{c} t\right)$. The amplifier has a gain factor, $k$, where the gain is set by a negative feedback loop from a power meter monitoring the output of the amplifier. The power meter determines the average power, $W$, at the output of the amplifier every $T_{\mathrm{s}}$ seconds, and sets the amplifier gain so that it is inversely proportional to a power transformation of $W$. Note that when the exponent $q$ is 0 , the amplification factor is 1 , and the amplifier is linear. The out- put of the amplifier is also sent to a discrimination circuit that has internal noise, $g(t)$, which serves to degrade the signal. It is assumed that this discrimination circuit has a range, before peak-clipping occurs, of $100 \mathrm{mV}$. Let us first consider how this amplifier would handle discrimination for different ranges of stimuli if the amplifier is assumed to be linear ( $q$ is set to 0 ), with the amplification factor, $k$, set by some higher order process.

Suppose that the three stimuli to be presented during an experiment have peak amplitudes of $17.5,35$, and $70 \mathrm{mV}$. Clearly, setting the value of $k$ to 1 maximizes the signal-to-noise ratios for all of the stimuli, but still produces no distortion in the discrimination circuit. In signal detection theory, for an ideal observer, the discriminability of pure tones in a background of constant-spectrumlevel noise will be directly proportional to their amplitude difference; that is, for $k=1, d^{\prime}=c\left(A_{j}-A_{i}\right)$, where $c$ is a proportionality constant. (In the present model it is assumed that the noise is introduced subsequent to the amplification stage, as shown in Figure 4.) Now let us see what happens to the discriminability of these signals when a sinusoidal signal with peak amplitude of $700 \mathrm{mV}$ is added to the set of possible stimuli. In order to avoid distortion, the amplification constant $k$ would have to be set to, at most, 0.14 . This, however, would reduce $d^{\prime}$ for comparisons among the three lowest tones by a factor of 


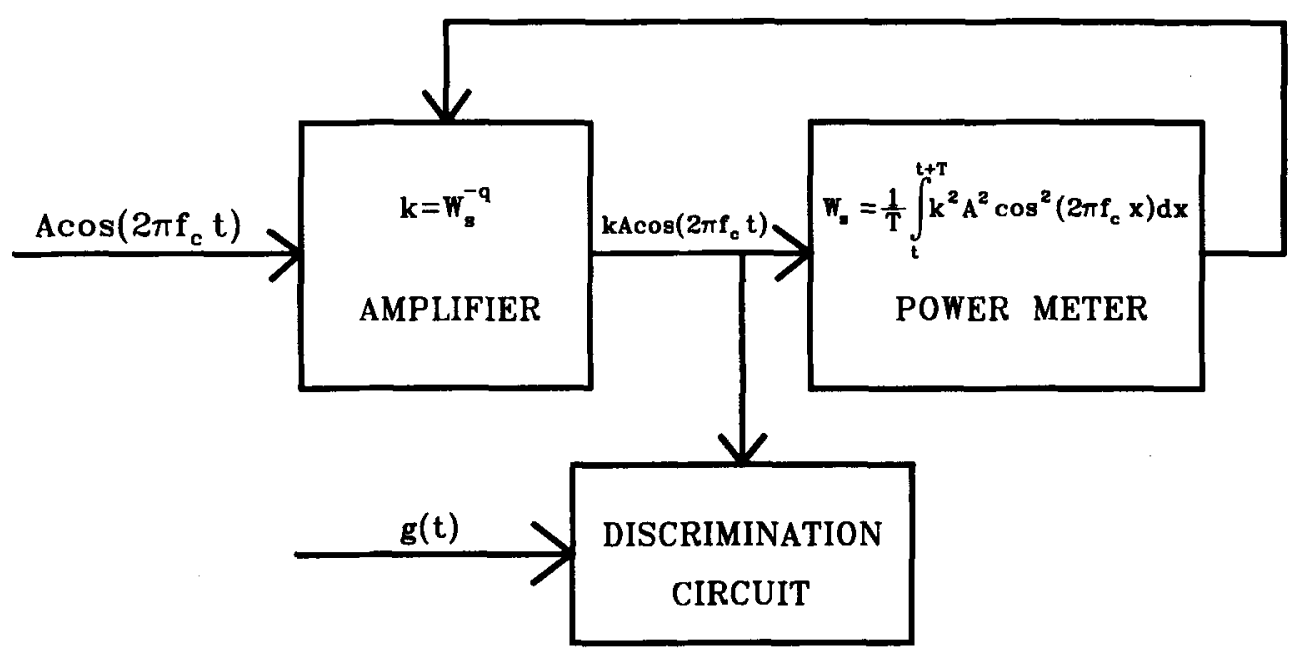

Figure 4. Diagram of a hypothetical nonlinear amplifier with feedback control. The output of the amplifier, as well as internal noise, $g(t)$, are routed to a discrimination circuit.

at least 7; that is, for $k=.14, d^{\prime}=.14 c\left(A_{j}-A_{i}\right)$. Large linear reductions in gain, therefore, dramatically reduce the discriminability of low-intensity tones.

Suppose, however, that instead of changing $k$ linearly, we allow its value to be determined by the negative feedback loop shown in Figure 4, with the value of $q=.3$. When the tone is first presented to the amplifier, there will be some fluctuation in the value of $k$ because of the feedback loop, but after about 10 cycles, the value of $k$ will have reached its equilibrium value; that is, there will no longer be any fluctuations in $k$ (see the Appendix). The equilibrium value, $k_{\mathrm{e}}$, for $q=.3$ is $k_{\mathrm{e}}=\left(A^{2} / 2\right)^{-.3 / 1.6}$. Therefore, once $k_{\mathrm{e}}$ has reached its equilibrium value, the amplitude of the signal going into the discrimination circuit would be $A^{\prime}=2^{1 / 2}\left(A / 2^{1 / 2}\right)^{1 / 1.6}$. Because $k$ is effectively constant after about 10 cycles of the signal, the output signal from the amplifier is an undistorted, attenuated version of the input signal. The values of $A^{\prime}$ for $A=17.5$, 35,70 , and $700 \mathrm{mV}$ are, respectively, $6.88,10.64,16.45$, and $70 \mathrm{mV}$. Because the value of $d^{\prime}$ is proportional to the differences in the $A^{\prime}$ values $\left[d^{\prime}=c\left(A_{j}^{\prime}-A_{i}^{\prime}\right)\right]$, a comparison of the linear amplifier with $k$ set to .14 for every tone, with the nonlinear amplifier in which the equilibrium value of $k$ varies with tonal amplitude, shows that the soft tones are more discriminable when the amplifier is nonlinear. Thus, when (1) the task is discrimination, (2) the potential amplitude range is large, (3) internal noise is added after the amplification stage, and (4) individual components in the information-processing flow are amplitude limited, the astute employment of nonlinear amplifiers in the information-processing flow will maximize the discriminability of soft tones.

In order for a nonlinear amplifier (like the one shown in Figure 4) to be able to maximize discriminability, the parameter, $q$, has to be set so that the tone with the highest expected amplitude will be sufficiently attenuated by the amplifier so that it does not exceed the input limitations of the discrimination circuit. Thus, the value of $q$ has to be set by higher order processes that take into account the range of tones that are likely to be encountered. There is a genuine reluctance to hypothesize this sort of top-down control of basic auditory processing among auditory theorists. However, if the loudness spacing among tones is changed by changing the range of tones, and this effect cannot be attributed to biases in the response system, then some sort of top-down control is indicated. The negative feedback circuit shown in Figure 4, with the value of $q$ as continuous and set by higher order processes, is one model of how such a process could work.

The reason that higher order processes are required is that the relations among the loudnesses of the stimuli in Condition $S$ were altered by adding loud stimuli to the set, as in Condition B. Therefore, modification of the nonlinear amplifier results from some process that keeps track of the recent loudness levels within an auditory filter. It seems unlikely that any peripheral mechanism could store and use information over that time period. Thus, even if the mechanism of the amplifier resides in the cochlea, its control must be central. A number of recent studies (see Hudspeth, 1989) have shown that the transduction process in the inner ear is highly nonlinear and rapidly adjustable. It is possible, therefore, that the nonlinear amplifier resides in the far periphery of the auditory system. If it does reside in the mechanisms of the transduction process, then according to our results, the parameters of this amplifier must be set or controlled by higher order processes that send instructions to the amplifier via descending efferent pathways. Such pathways clearly exist, but whether they are capable of modulating the nonlinearities in the transduction process is unknown. Moreover, 
we cannot conclude from our experiments that the nonlinear process is actually located that far into the periphery. We can only conclude that it must occur somewhere in the auditory processing system.

The existence of such a nonlinear amplifier has implications for intensity-increment thresholds. Specifically, intensity-increment thresholds for low-intensity sounds should be smaller when tested in a sound context containing only low-intensity sounds than when tested in a context containing some very high-intensity sounds. However, intensity-increment thresholds for high-intensity tones should be independent of the presence or absence of some low-intensity tones into the overall sound context. Experiments are currently under way in order to test this notion.

\section{REFERENCES}

Algom, D., \& Marks, L. E. (1990). Range and regression, loudness scales, and loudness processing: Toward a context-bound psychophysics. Journal of Experimental Psychology: Human Perception \& Performance, 16, 706-727.

BissetT, R. J., \& SchNeider, B. (1991). Spatial and conjoint models based on pairwise comparisons of dissimilarities and combined effects: Complete and incomplete designs. Psychometrika, 56, 685-698.

FAGOT, R. F. (1959). A model of ordered metric scaling by comparisons of intervals. Psychometrika, 24, 157-168.

Fuortes, M. G. F., \& HodGkIN, H. L. (1964). Changes in time scale and sensitivity in the ommatidia of Limulus. Journal of Physiology, 172, 239-263.

GeisLER, C. D. (1974). Hypothesis on the function of the crossed olivocochlear bundle. Journal of the Acoustical Society of America, 56, 1908-1909.

HudSPETH, A. J. (1989). How the ear's works work. Nature, 341, 397-404.

KIM, D. O. (1984). Functional roles of the inner- and outer-hair-cell subsystems in the cochlea and brainstem. In C. I. Berlin (Ed.), Hearing science: Recent advances (pp. 241-262). San Diego: College-Hill Press.

MARKs, L. E. (1972). Visual brightness: Some applications of a model. Vision Research, 12, 1409-1423.

MARKS, L. E. (1979). A theory of loudness and loudness judgments. Psychological Review, 86, 256-285.

MARKS, L. E. (1988). Magnitude estimation and sensory matching. Perception \& Psychophysics, 43, 511-525.

MARKs, L. E. (1992). The contingency of perceptual processing: Context modifies equal-loudness relations. Psychological Science, 3 , 285-291.

Marks, L. E., Szczesiul, R., \& OHLotT, P. (1986). On the crossmodal perception of intensity. Journal of Experimental Psychology: Human Perception \& Performance, 12, 517-534.

MARKS, L. E., \& WARNER, E. (1991). Slippery context effect and critical bands. Journal of Experimental Psychology: Human Perception \& Performance, 17, 986-996.

Poulton, E. C. (1968). The new psychophysics: Six models for magnitude estimation. Psychological Bulletin, 69, 1-19.

RoBrnson, G. H. (1976). Biasing power law exponents by magnitude estimation instructions. Perception \& Psychophysics, 19, 80-84.

SCHNEIDER, B. (1980). Individual loudness functions determined from direct comparisons of loudness intervals. Perception \& Psychophysics, 28, 493-503.

Schneider, B., \& PARKer, S. (1990). Does stimulus context affect loudness or only loudness judgments? Perception \& Psychophysics, 48, $409-418$.

SChNeIder, B., PARKer, S., \& STEIN, D. (1974). Measurement of loudness using direct comparisons of sensory intervals. Journal of Mathematical Psychology, 11, 259-273.
Schneider, B., Parker, S., Valenti, M., Farrell, G., \& Kanow, G. (1978). Response bias in category and magnitude estimation of difference and similarity for loudness and pitch. Journal of Experimental Psychology: Human Perception \& Performance, 4, 483-496.

SPERling, G., \& SondHI, M. M. (1968). Model for visual luminance discrimination and flicker detection. Journal of the Optical Society of America, 58, 1133-1145.

Stevens, S. S. (1957). On the psychophysical law. Psychological Review, 64, 153-181.

STEvens, S. S. (1971). Issues in psychophysical measurement. Psychological Review, 78, 426-450.

STEVENS, S. S., \& GALANTER, E. (1957). Ratio scales and category scales for a dozen perceptual continua. Journal of Experimental Psychology, 54, 377-411.

Stevens, S. S., \& Greenbaum, H. B. (1966). Regression effect in psychophysical judgment. Perception \& Psychophysics, 1, 439-446. TEGHTSOONiAN, R. (1973). Range effects in psychophysical scaling and a revision of Stevens' law. American Journal of Psychology, 86, 3-27. ZWISLOCKI, J. J., \& GoODMAN, D. A. (1980). Absolute scaling of sensory magnitudes: A validation. Perception \& Psychophysics, 28, 28-38.

\section{NOTES}

1. Any single set of projection values represents interval scale measurement of loudness and so can be specified uniquely only up to an affine transformation-that is, up to multiplication by a constant and addition of a constant. Thus, for example, in Figure 2 the locations of the triangles can be shifted left or right (or up or down) by any fixed number of units, without changing the predicted outcomes of the paired comparison judgments. Shifts along the $x$-axis, however, do affect the estimated value of the exponent in the power function relating the loudness scales in the two conditions. In particular, shifting the loudness projections to the right (larger additive constant for the projections shown along the abscissa) increases the size of the fitted exponent for plots showing positive acceleration. To avoid exaggerating the degree of departure from linearity, we chose to adjust the additive constant in each set so that the lowest loudness level was assigned a value of zero, which had the consequence of minimizing the size of the estimated exponent.

Although additive shifts affect the precise value of the estimated exponents, their effects are limited. In particular, the estimated exponent will exceed 1.0 for all additive shifts, will be 1.0 for all additive shifts, or will be less than 1.0 for all additive shifts. A geometric interpretation of this is that affine transformation (multiplication by a constant or addition by a constant) cannot make a straight line curve, cannot make a curved line straight, and cannot transform one type of curvature (upward or downward) into the other. Moreover, identical shifts do not change the rank order of the exponents because shifts do not change the degree of curvature.

2 . It should be noted that experiments involving judgments of differences typically result in lower exponents than those involving judgments of magnitudes or judgments of ratios (Marks, 1979). This issue is discussed more fully in Schneider (1980).

\section{APPENDIX}

In the circuit in Figure 4, the input to the amplifier is a sinusoid whose frequency is equal to the center frequency, $f_{c}$, of the auditory filter. If $k$ is the amplification factor, then the output is simply $k$ times the input. The output of the amplifier is monitored by a power meter. The power meter computes the power coming from the amplifier every $T$ seconds, where $T$ is the period of a signal whose frequency is $f_{\mathrm{c}}$. Therefore, the power in a $T$ second period is

$$
W_{s}=\left(\frac{1}{T}\right)^{t+T} \int_{i}^{2} k^{2} \cos ^{2}\left(2 \pi f_{\mathrm{c}} x\right) d x=k^{2}\left(\frac{A^{2}}{2}\right) .
$$

Every $T$ seconds the amplifier looks at the current value of $W_{\mathrm{s}}$ and changes its amplification factor to $k=W_{\mathrm{s}}^{-q}$. In other 
words, the circuit computes the power, $W_{s}$, at the output of the amplifier every $T$ seconds, and sets the value of the amplification factor so that it is inversely proportional to the $q$ th power of $W_{s}$. After some initial oscillation that will occur when the signal is first turned on, the amplification factor will reach an equilibrium value, $k_{\mathrm{e}}$. The equilibrium value occurs when

$$
\begin{gathered}
k_{\mathrm{e}}=\left[k_{\mathrm{e}}^{2}\left(\frac{A^{2}}{2}\right)\right]^{-q} \\
k_{\mathrm{e}}^{1+2 q}=\left(\frac{A^{2}}{2}\right)^{-q} \\
k_{\mathrm{e}}=\left(\frac{A^{2}}{2}\right)^{-q /(1+2 q)} .
\end{gathered}
$$

Equation A2 shows that once $k$ becomes equal to $k_{\mathrm{e}}$, there will be no further changes in $k$. In determining the conditions under which $k$ will converge to $k_{\mathrm{e}}$, we will first show that $k$ will not converge to $k_{\mathrm{e}}$ if $q \geq .5$. Let $k_{n}$ represent the value of $k$ computed on the $n$th sample. It follows that

$$
\begin{gathered}
k_{n+1}=\left[k_{n}^{2}\left(\frac{A^{2}}{2}\right)\right]^{-q} \\
k_{n+1}=k_{n}^{-2 q}\left(\frac{A^{2}}{2}\right)^{-q} \\
k_{n+2}=\left[k_{n+1}^{2}\left(\frac{A^{2}}{2}\right)\right]^{-q}=k_{n}^{4 q^{2}}\left(\frac{A^{2}}{2}\right)^{2 q^{2}-q} \\
\frac{k_{n+2}}{k_{n}}=k_{n}^{4 q^{2}-1}\left(\frac{A^{2}}{2}\right)^{2 q^{2}-q} .
\end{gathered}
$$

Note that if $q>.5$, then for at least some values of $k_{n}$ and $A^{2} / 2$, convergence to the equilibrium point will not occur because the ratio of $k_{n+2} / k_{n}$ will always be greater than 1 . Suppose that both $A^{2} / 2$ and $k_{n}$ are greater than 1 , and $q>.5$. Then $k$ would continue to increase indefinitely because this ratio would always be greater than 1 . Suppose, however, that $q<.5$, and $k_{n}$ differs from the equilibrium point by a factor, $a$. So,

$$
k_{n}=a\left(\frac{A^{2}}{2}\right)^{-q /(1+2 q)}
$$

Then the ratio becomes

$$
\frac{k_{n+2}}{k_{n}}=\left[a\left(\frac{A^{2}}{2}\right)^{-q /(1+2 q)}\right]^{4 q^{2}-1}\left(\frac{A^{2}}{2}\right)^{2 q^{2}-q}=a^{4 q^{2}-1}
$$

Note that if $a<1$, and $q<.5$, then $k_{n}$ is less than the equilibrium value and $k_{n+2}$ is between $k_{n}$ and the equilibrium point. If $a>1$ and $q<.5$, then $k_{n+2}<k_{n}$, but greater than the equilibrium point. Therefore, the circuit will converge for $0 \leq q<.5$. The rate of convergence can be determined by simulating the circuit, starting with different initial values of $k$. The results of the simulation for $q=.3$, the value used for the example in the text, showed that the equilibrium value was essentially reached in 10 steps. Because each step is a period of the waveform, an amplifier such as this would converge on the equilibrium point within 10 cycles of signal onset.

Note that the steady-state output of the amplifier for a constant input will be

$$
\begin{aligned}
k_{\mathrm{e}} A \cos 2 \pi f_{\mathrm{c}} t & =\left(\frac{A}{\sqrt{2}}\right)^{-2 q /(1+2 q)} \sqrt{2}\left(\frac{A}{\sqrt{2}}\right) \cos 2 \pi f_{\mathrm{c}} t \\
& =\sqrt{2}\left(\frac{A}{\sqrt{2}}\right)^{1 /(1+2 q)} \cos 2 \pi f_{\mathrm{c}} t
\end{aligned}
$$

Therefore, the steady-state output of the circuit will be a power function of its input with the exponent equal to $1 /(1+2 q)$. For the value of $q$ selected for the example in the text, the exponent would be approximately .63 .

(Manuscript received July 23, 1993; revision accepted for publication December 10, 1993.) 